\title{
Non-Isothermal Processes and Reactions- Summery on Research and Investigations
}

\author{
Sunil Jayant Kulkarni \\ Chemical Engineering Department \\ Datta Meghe College of Engineering \\ Airoli, Navi Mumbai, Maharashtra, India
}

\begin{abstract}
An adiabatic process is one that occurs without transfer of heat or matter between a thermodynamic system and its surroundings. Chemical reaction engineering deals with determining kinetics, mechanism and rates of the reactions. The reactions are classified as heterogeneous and homogeneous. Heterogeneous reactions are either catalytic or non-catalytic. Catalytic reactions are very important and are mainly used in petroleum and petrochemical industries. Current review summarizes research and studies on non-isothermal processes and reactions.
\end{abstract}

Keywords: Heat effects, temperature, diffusion, catalysis, degradation, mechanism.

\section{INTRODUCTION}

Temperature remains constant in isothermal process. An adiabatic process is one that occurs without transfer of heat or matter between a thermodynamic system and its surroundings. Chemical reaction engineering deals with determining kinetics, mechanism and rates of the reactions. The reactions are classified as heterogeneous and homogeneous. Heterogeneous reactions are either catalytic or noncatalytic. Catalytic reactions are very important and are mainly used in petroleum and petrochemical industries[1-4].Reactive adsorption is evolving field in catalyst applications[5-7].Recovery of flue gases involves heterogeneous reactions. Coal desulphurization includes many heterogeneous catalytic reactions with temperature effects [8-12]. In isothermal reactors, we need energy balance to calculate heat duty. In non-isothermal reactors heat effects are more important. Current review summarizes research and studies on non-isothermal processes and reactions.

\section{Non Isothermal Processes and Reactions- ReSEarCH AND InVEstigations}

Georgieva et. al. investigated non-isothermal kinetics of thermal degradation of Chitosan [13]. According to them, Chitosan is the second most abundant nitrogen containing biopolymer in nature. It finds application in biotechnology, biomedicine, food industries, and cosmetics. It absorbs a number of metal ions as its amino groups. According to the literature, hydroxyl, carboxyl, sulfate, phosphate, and amino groups on the Chitosan are responsible for metal binding and sorption of dyes and pigments. According to their studies, the selection of proper mechanism for the process is important factor in obtaining proper results. They observed that Chitosan has excellent properties such as hydrophilicity, biocompatibility, biodegradability, antibacterial, non-toxicity, adsorption application. Lim and Chew found that considerable variation exists in the kinetics constants for wood pyrolysis [14].They studied compensation effect to seek a logical explanation for the observed variations of the apparently large and differed values of activation energy and pre-exponential factors. They analyzed the transition kinetics for exothermic transition using differential scanning calorimetry (DSC). Ramli carried out investigation on modeling and control for composition of non-isothermal CSTR by using fuzzy [15]. It was shown that by using the fuzzy logic controller, there was a small integral absolute error. According to their results, the fuzzy logic gives the most favorable results. They concluded that it gives benefits in the development of industrial process control system. Khachani et.al. determined the non-isothermal kinetics of dehydroxylation of $\mathrm{Ca}(\mathrm{OH})_{2}[16]$. They carried out investigation in dynamic helium atmosphere using TG, DTG, DTA and XRD techniques at different heating rates. According to their results, dehydroxylation process occurred predominantly by an irreversible major step. They established the appropriate reaction model characterizing the process by applying the 
Malek's procedure. Saad et.al. developed an improved computational method for non-isothermal resin transfer molding simulation [17]. In their research they developed an improved computational approach based on finite element method coupled with control volume approach. They proposed a model which provided smoother flow fronts and reduced the error in the pressure and temperature pattern that plagued the conventional fixed grid methods.

Zarateet.al. carried out investigation on non-isothermal reaction-diffusion systems[18].They considered a simple reaction-diffusion system, namely a binary fluid mixture with an associationdissociation reaction. They studied fluctuations at hydrodynamic spatiotemporal scales due to a temperature gradient. Also they reviewed fluctuating hydrodynamics when the system was in global equilibrium. They predicted an enhancement of the intensity of concentration fluctuations in the presence of a temperature gradient. According to them, non-equilibrium fluctuations may be a good motivation to perform this very large-scale molecular simulations. Ke et.al. studied non-isothermal kinetics of styrene butadiene styrene asphalt combustion[19]. According to them, the combustion process of SBS asphalt can be divided by Gaussian peak fitting into three main stages: oil content release, resin pyrolysis, and asphalting and char combustion. They observed that the sphere phase boundary reaction model, the second order chemical reaction model, nucleation, and its subsequent growth model explained the three stages of asphalt combustion. Georgieva et.al. Studied nonisothermal degradation kinetics of $\mathrm{CaCO}_{3}$ [20].They carried out investigation on two varieties of calcium carbonate, namely, analytical reagent-grade and in situ product from thermal degradation of calcium oxalate monohydrate. They found that the results were dependent on the selection of proper mechanism function for the process. According to them, the isoconversion calculation procedure was more appropriate.

Maitra et.al. studied kinetics of non-isothermal decomposition for magnesite[21].They used thermogravimetric analysis under non-isothermal condition. They analyzed different kinetic functions with the experimental data to ascertain the decomposition mechanism of magnesium carbonate. They found that the decomposition reaction followed a contracting sphere kinetic mechanism. Antonelli and Astolfi investigated methodologies and tools from Lyapunov theory in the design of bounded control laws [22]. They used these laws for the temperature stabilization of a class of continuous stirred tank reactors with exothermic or endothermic reactions. They observed that these controllers yielded global asymptotic stability. They do not require precise knowledge of the system parameters. Reza and Ali carried out an investigation on kinetic aspects of non-isothermal oxidation of molybdenite[23]. They studied kinetic analysis of the reaction. They found that the rate of the reaction of molybdenite oxidation was rate determining step. In chemically controlled regime, the popular model-fitting indicated excellent fit for non-isothermal data. Mallik and Rout investigated the growth kinetics of low temperature sono-electrochemically deposited $\mathrm{Cu}$ thin films [24]. The studies were carried out under non-isothermal conditions. They used a differential scanning calorimetric (DSC) technique in their investigation. They found that, as film temperature was reduced, there was a transition from abnormal growth to normal growth. Hoang et.al. derived nonlinear control laws for the stabilization of a large class of CSTRs[25].In their investigation, they considered a liquid mixture with constant volume. There was the constraint on the volume. It was insured by perfect regulation of the outlet flow of the CSTR. It was shown by them that the availability function can be split into two parts. According to them, one corresponds to the mixing term and depends on mole numbers only and the other depends on both temperature and mole numbers. Joraid et.al. Performed differential scanning calorimetry (DSC) under nonisothermal conditions at various heating rates [26].They carried out studies for glassy Se made by high-energy ball milling. In their investigation, they compared three methods namely the ball milling technique and the melt-quenching and thin-film techniques. They calculated thermal stability of the Se glasses by calculation of the temperature difference $(\mathrm{Tc}-\mathrm{Tg})$ and the S-parameter. Their investigations indicated that the glasses formed using the thin film technique were the most stable.

Jian-he et.al. investigated non-isothermal decomposition kinetics of $\mathrm{LiClO}_{4}$ in flow $\mathrm{N}_{2}$ atmosphere[27]. They considered the activation energies calculated with the Friedman method as the initial values and carried out non-linear regression. The values of activation energies calculated from the isothermal method were in agreement with kinetic data. Sediawan and Megawati used Monte Carlo simulation to study non-isothermal acid hydrolysis of lignocellulosic material in ethanol production [28]. According to them, high temperature hydrolysis is one of important steps to produce lignocellulosic ethanol. They explored the possibility of use of Monte Carlo simulation to deeply 
study the progress of non-isothermal hydrolysis. They compared these results with the simulation results obtained from kinetics. They obtained good agreement between results. Jamili-Shirvan et.al. investigated thermal behavior and non-isothermal crystallization [29].They carried out studies for bulk metallic glass. Scanning calorimetry method was used by them for the analysis. At lower heating rates four exothermic and three endothermic peaks were observed by them.

$\mathrm{Xu}$ and Pruess investigated non-isothermal fluid flow and reactive geochemical transport in variably saturated fractured rocks [30]. They presented methods for modeling geochemical systems. In their paper, Talkner et.al. described a Gaussian binary collision theory[31]. It included all three aspects namely radioactive decay, nonradioactive decay and relaxation. Also they presented an analytic solution for the Gaussian master equation. They observed that pressure induced decay is an experimental signature of an initial cold distribution of reactants. In their investigation, Gupta et.al. presenteda non-isothermal model for the fluid catalytic cracking riser reactor [32].They used the difference in the heats of combustion of products and reactants for calculating heat of cracking. They used Lee-Kessler's correlations for estimating heat capacities of product and reactant pseudocomponents as a function of local temperature. They also incorporated the effect of local temperature on the kinetic constants. They concluded that the proposed model was capable of predicting the products' yields, velocities of cluster phase and gas phase, riser temperature, and heat of cracking.

\section{CONClusion}

Chemical reaction engineering deals with determining kinetics, mechanism and rates of the reactions. The reactions are classified as heterogeneous and homogeneous. Heterogeneous reactions are either catalytic or non-catalytic. Catalytic reactions are very important and are mainly used in petroleum and petrochemical industries. Reactive adsorption is evolving field in catalyst applications. Recovery of flue gases involves heterogeneous reactions. Coal desulphurization includes many heterogeneous catalytic reactions with temperature effects. In isothermal reactors, we need energy balance to calculate heat duty. In non-isothermal reactors heat effects are more important.

\section{REFERENCES}

[1] Morris D. Argyle and Calvin H. Bartholomew (2015), "Heterogeneous Catalyst Deactivation and Regeneration: A Review", Catalysts, 5, 145-269.

[2] Sunil J. Kulkarni(2015), "A Review on Studies and Research on Catalysts with Emphasis on Catalyst Deactivation", International Journal of Research and Review, 2(10), 610-614

[3] Majid Saidi, Navid Mostoufi, Rahmat Sotudehgharebagh (2011), "Modeling And Simulation Of Continuous Catalytic Regeneration (CCR) Process", International Journal Of Applied Engineering Research, Dindigul, 2(1), 115-124.

[4] Sunil Jayant Kulkarni(2016), "A Review on Petroleum Refining and Petrochemical Processes with Special Emphasis on Catalysts and Flue Gas Treatment Technology", International Journal of Petroleum and Petrochemical Engineering, 2(1), 1-5.

[5] Sunil Jayant Kulkarni(2015), "Advancements, Research and Challenges in Reactive Adsorption: A Review", International Journal of Research, 2(1),477-480.

[6] Sabine Goldberg, Louise J. Criscenti, David R. Turner, James A. Davis, and Kirk J. Cantrell(2007), "Adsorption-Desorption Processes in Subsurface Reactive Transport Modeling", www.vadosezonejournal.org., 6, (3), 407-435.

[7] Igor Bezverkhyy, Andrey Ryzhikov, Geoffroy Gadacz, Jean-Pierre Bellat(2008), "Kinetics of thiophene reactive adsorption on Ni/SiO2and Ni/ZnO", Catalysis Today,130, 199-205, 2008.

[8] A.M. Saeid, D.A. Butcher and N.A. Rowson(1993), "Coal Desulphurization and Ash Removal in Intensified Magnetic Fields", Magnetic and Electrical Separation, 1993, 4, 107-116, 1993.

[9] Ehsani, Mohammad Reza; Eghbali, Farahnaz(2007), "Reduction of Sulfur and Ash from Tabas Coal by Froth Flotation", Iran. J. Chem. Chem. Eng., 26(2), 35-40.

[10] Tian Yingzhong, Zhang Qin, Tang Yun, Qiu Yueqin, Liu Zhihong, He Tin, Zhao Peiliang, Huang Xiaofen, Shi Ren, Tiancun Xiao(2010), "Float-Sink Desulfurization Of High-Sulphur Coal From Puan County, Guizhou Province, PRC", Petroleum \& Coal, 52 (4) 249-253.

[11] Kulkarni SJ.(20160, "Research and studies on coal desulphurization", Int J Res Rev., 3(6),56-58.

[12] Sunil Jayant Kulkarni(2016), "Role of Adsorption in Petroleum Industries and Refineries", International Journal of Petroleum and Petrochemical Engineering, 2(1), 1-4. 
[13] VelyanaGeorgieva, DilyanaZvezdova And LyubomirVlaev(2012), "Non-Isothermal Kinetics Of Thermal Degradation Of Chitosan", Chemistry Central Journal, 6(81), 1-10.

[14] Siew Mei Lim And Michael Yit Lin Chew (2005), "Compensation Effects In The NonIsothermal Pyrolysis Of Wood", Copyright (C) 2005 International Association For Fire Safety Science Fire Safety Science-Proceedings Of The Eighth International Symposium, 1109-1120.

[15] Nasser Mohamed Ramli(2016), "Modelling And Control For Composition Of Non-Isothermal Cstr By Using Fuzzy", International Journal Of Engineering Sciences \& Research, 5(9),112-116.

[16] M. Khachani, A. El Hamidi, M. Halim, S. Arsalane (2014), "Non-Isothermal Kinetic And Thermodynamic Studies Of The Dehydroxylation Process Of Synthetic Calcium Hydroxide $\mathrm{Ca}(\mathrm{OH})_{2}$ ", J. Mater. Environ. Sci., 5 (2),615-624

[17] AouatifSaadA, AdilEchchelhA, Mohammed HattabiB, And Mohammed El Ganaoui (2011), "An Improved Computational Method For Non-Isothermal Resin Transfer Moulding Simulation”, Thermal Science, 15(2), S275-S289.

[18] Jose M. Ortiz De Zarate(2007), "Concentration Fluctuations In Nonisothermal ReactionDiffusion Systems", The Journal Of Chemical Physics, 127, 1-4.

[19] Wu Ke, Zhu Kai, Han Jun,Wang Jin-Chang, Huang Zhi-Yi, And Liang Pei(2013), "NonIsothermal Kinetics Of Styrene Butadiene Styrene Asphalt Combustion", Chin. Phys. B., 22(6), $1-6$.

[20] Velyana Georgieva, Lyubomir Vlaev, And Kalinka Gyurova (2013), "Non-Isothermal Degradation Kinetics Of $\mathrm{CaCO}_{3}$ From Different Origin”,Hindawi Publishing Corporation Journal Of Chemistry, Volume 2013, Article Id 872981, 12 Pages.

[21] S. Maitra, S. Mukherjee, N. Saha, J. Pramanik (2007), "Non-Isothermal Decomposition Kinetics Of Magnesite",Ceramica, 53, 284-287.

[22] R. Antonelli, A. Astolfi(2003), "Continuous Stirred Tank Reactors: Easy To Stabilise?", Automatica, 39,1817 - 1827.

[23] Ebrahimi Kahrizsangi, Reza, Abbasi, Mohammad Hasan; Saidi, Ali (2007), "Model-Fitting Approach To Kinetic Analysis Of Non-Isothermal Oxidation Of Molybdenite", Iran. J. Chem. Chem. Eng., 26(2), 119-123.

[24] A.Mallik 1 And S. Rout(2011), "A Study On Ex-Situ Non-Isothermal Grain Growth Behavior Of Sono-Electrochemically Deposited Cu Thin Films", International Journal Of Materials Science, 6(1), 77-88.

[25] Ha Hoang, Fran_CoiseCouenne, Christian Jallut, Yann Le Gorrec(2012), "Lyapunov-Based Control Of Non Isothermal Continuous Stirred Tank Reactors Using Irreversible Thermodynamics", Journal Of Process Control.,22(2), 412-422, <10.1016 /J.Procont. 2011 $.12 .007>$.<Hal-00803656>

[26] A.A. Joraid, S.N. Alamri, A.A. Abu-Sehly 1, M. Benghanem(2012), "Nonisothermal Crystallisation Kinetics Of Amorphous Selenium Prepared By High-Energy Ball Milling: A Comparison With The Melt-Quenching And Thin-Film Techniques", Journal Of Non-Crystalline Solids, 358, 1268-1273.

[27] Hong Jian-He,Song Li, Zhou Xin-Wen, DiakiteKahirou And Zhang Ke-Li(2010), "Non-Isothermal Decomposition Mechanism And Kinetics Of Liclo4 In Nitrogen", Chem. Res. Chinese Universities, 26(2), 300-303.

[28] Wahyudi Budi Sediawan, And Megawati(2013), "Monte Carlo Simulation To Study Non-Isothermal Acid Hydrolysis Of Lignocelluloses Material In Ethanol Production", International Journal Of Chemical, Environmental \& Biological Sciences,1(3),7-11.

[29] Zahra Jamili-Shirvan A,B, Mohsen Haddad-Sabzevar A, JalilVahdati-Khaki A, Ke-Fu Yaob(2016), "Thermal Behavior And Non-Isothermal Crystallization Kinetics Of (Ti41zr25be28fe6)93cu7 Bulk Metallic Glass", Journal Of Non-Crystalline Solids,447, 156-166.

[30] TianfuXu And KarstenPruess (2001), "Modeling Multiphase Non-Isothermal Fluid Flow And Reactive Geochemical Transport In Variably Saturated Fractured Rocks: Methodology", American Journal Of Science, 301, 16-33.

[31] Peter Talkner A, Eli Pollak B, Alexander M. Berezhkovskii (1998), "Binary Collision Theory For Thermal And Nonisothermal Relaxation And Reaction Of Polyatomic Molecules", Chemical Physics, 235, 131-146.

[32] Raj Kumar Gupta, Vineet Kumar, And V.K. Srivastava (2007), "Modeling Of Non-Isothermal Fluid Catalytic Cracking Riser Reactor", Proceedings Of The World Congress On Engineering And Computer Science, WCECS 2007, October 24-26, 2007, San Francisco, USA, 1-6. 